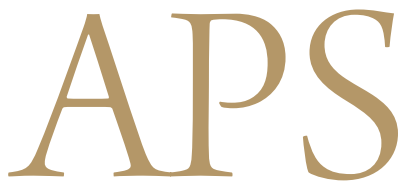

Archives of Plastic Surgery

\title{
Comparison of the Multidetector-row Computed Tomographic Angiography Axial and Coronal Planes' Usefulness for Detecting Thoracodorsal Artery Perforators
}

\author{
Jong Gyu Kim, Soo Hyang Lee \\ Department of Plastic and Reconstructive Surgery, Ilsan Paik Hospital, Inje University College of Medicine, Goyang, Korea
}

Background During the planning of a thoracodorsal artery perforator (TDAP) free flap, preoperative multidetector-row computed tomographic (MDCT) angiography is valuable for predicting the locations of perforators. However, CT-based perforator mapping of the thoracodorsal artery is not easy because of its small diameter. Thus, we evaluated 1-mm-thick MDCT images in multiple planes to search for reliable perforators accurately.

Methods Between July 2010 and October 2011, 19 consecutive patients (13 males, 6 females) who underwent MDCT prior to TDAP free flap operations were enrolled in this study. Patients ranged in age from 10 to 75 years (mean, 39.3 years). MDCT images were acquired at a thickness of $1 \mathrm{~mm}$ in the axial, coronal, and sagittal planes.

Results The thoracodorsal artery perforators were detected in all 19 cases. The reliable perforators originating from the descending branch were found in 14 cases, of which 6 had transverse branches. The former were well identified in the coronal view, and the latter in the axial view. The location of the most reliable perforators on MDCT images corresponded well with the surgical findings.

Conclusions Though MDCT has been widely used in performing the abdominal perforator free flap for detecting reliable perforating vessels, it is not popular in the TDAP free flap. The results of this study suggest that multiple planes of MDCT may increase the probability of detecting the most reliable perforators, along with decreasing the probability of missing available vessels.

Keywords Angiography / Free tissue flaps / Multidetector computed tomography
Correspondence: Soo Hyang Lee Department of Plastic and Reconstructive Surgery, Ilsan Paik Hospital, Inje University College of Medicine, 170 Juhwa-ro, Ilsanseo-gu, Goyang 411-706, Korea Tel: +82-31-910-7320 Fax: +82-31-910-7814 E-mail: shyanglee@naver.com

This article was presented at the the 69th Annual Conference of the Korean Society of Plastic and Reconstructive Surgeons on November 11-13, 2011 in Seoul, Korea.

No potential conflict of interest relevant to this article was reported.

\section{INTRODUCTION}

During the planning of the thoracodorsal artery perforator (TDAP) free flap, preoperative multidetector-row computed tomographic angiography (MDCT) is valuable for predict- ing the locations of perforators [1]. However, the computed tomography (CT)-based mapping of the thoracodorsal artery perforators is not easy because of their relatively small diameters. Applying anatomical landmarks for the skin perforators of the thoracodorsal artery could be an alternative, but uncertainty due

Copyright $\odot 2012$ The Korean Society of Plastic and Reconstructive Surgeons

This is an Open Access article distributed under the terms of the Creative Commons Attribution Non-Commercial License (http://creativecommons.org/

licenses/by-nc/3.0/) which permits unrestricted non-commercial use, distribution, and reproduction in any medium, provided the original work is properly cited.

www.e-aps.org 
to unexpected anatomical variations is a problem [2]. Furthermore, Doppler flowmetry has an unacceptably low sensitivity and low positive predictive values [3].

Therefore, we have taken 1-mm thick MDCT images in multiple planes to make better use of the MDCT in planning the TDAP free flap, and reviewed the coronal, sagittal, and axial images in order to accurately detect reliable perforators.

\section{METHODS}

Between July 2010 and October 2011, a total of 19 patients (13 males, 6 females) who underwent MDCT prior to TDAP free flap were enrolled in this study. The mean age of the patients was 39.3 years (range, 10 to 75 years). Of the 19 patients, 17 who had sustained trauma to their extremities and 2 patients who had undergone extensive tumor resection of the scalp and sole received free flaps.

We performed imaging studies preoperatively using a 320-slice MDCT (Aquilion ONE, Toshiba Medical, Tokyo, Japan) with the following parameters: $120 \mathrm{kVp}, 450 \mathrm{~mA}$ (0.6-second gantry rotation time), $100 \times 0.5 \mathrm{~mm}$ detector configuration, $0.5-\mathrm{mm}$ beam width, 0.87 beam pitch, $13.75-\mathrm{mm}$ table feed per rotation, and $512 \times 512$ matrix. For all of the scans, $150 \mathrm{~mL}$ of $300 \mathrm{mg} /$ $\mathrm{mL}$ nonionic contrast (Pamiray 370, Dongkuk Pharmaceutical, Seoul, Korea) was intravenously administered at a rate of 3.5 $\mathrm{mL} / \mathrm{sec}$ through an 18-gauge catheter inserted into the antecubital vein.
Patients were placed in the lateral decubitus or supine position with their shoulders extended during the MDCT. Scans ranged from the shoulder to the umbilicus. The raw data were reconstructed in multiple planes with a slice width of $1 \mathrm{~mm}$ and a reconstruction interval of $0.8 \mathrm{~mm}$. The data were also reconstructed in three-dimensional images, which helped assess the individual variations of the vascular anatomy accurately.

A single surgeon detected and assessed the reliable perforators. The courses beneath the deep fascia and perforating points were noted and marked on the MDCT images. At the time of surgery, the MDCT results were compared with the surgical findings.

\section{RESULTS}

In all cases, the thoracodorsal artery perforators were found using MDCT with a satisfactory concordance between the radiologic images and operative findings. The preoperative surgical plans based on the MDCT images were successfully executed without any problems. The descending branch of the thoracodorsal artery had large and reliable perforating vessels in 14 patients (Table 1). Ten perforating vessels were well identified in the coronal and axial views of MDCT, and 4 perforators from the descending branch were detected in only the coronal view (Figs. 1,2). Six cases had perforators with a significant diameter originating from the transverse branch based on MDCT images. These perforators were found in the axial view (Fig. 3). One case had both descending and transverse perforators (Fig. 4).

\section{Table 1. Patient clinical information}

\begin{tabular}{|c|c|c|c|c|c|}
\hline Patient & Age/Sex & Cause & Recipient site & $\begin{array}{l}\text { Branch of the } \\
\text { thoracodorsal artery }\end{array}$ & MDCT images \\
\hline 1 & $14 / \mathrm{M}$ & Trauma & Dorsum of foot & Descending & Coronal \& axial \\
\hline 2 & $75 / F$ & Neoplasm & Scalp & Descending & Coronal \& axial \\
\hline 3 & $37 / F$ & Trauma & Lower leg & Descending & Coronal \\
\hline 4 & 40/M & Trauma & Lower leg & Transverse & Axial \\
\hline 5 & $54 / \mathrm{M}$ & Trauma & Lower leg & Descending & Coronal \\
\hline 6 & $23 / F$ & Trauma & Lower leg & Descending & Coronal \& axial \\
\hline 7 & $10 / F$ & Trauma & Dorsum of foot & Descending & Coronal \& axial \\
\hline 8 & $33 / M$ & Trauma & Elbow & Descending & Coronal \& axial \\
\hline 9 & $47 / M$ & Trauma & Lower leg & Transverse & Axial \\
\hline 10 & $37 / M$ & Trauma & Lower leg & Transverse & Axial \\
\hline 11 & 49/M & Trauma & Lower leg & $\begin{array}{l}\text { Descending } \\
\text { Transverse }\end{array}$ & $\begin{array}{l}\text { Coronal } \\
\text { Axial }\end{array}$ \\
\hline 12 & $42 / F$ & Trauma & Dorsum of foot & Transverse & Axial \\
\hline 13 & $24 / M$ & Trauma & Lower leg & Descending & Coronal \& axial \\
\hline 14 & $54 / \mathrm{M}$ & Trauma & Lower leg & Descending & Coronal \\
\hline 15 & $48 / M$ & Trauma & Lower leg & Descending & Coronal \& axial \\
\hline 16 & $34 / F$ & Trauma & Lower leg & Transverse & Axial \\
\hline 17 & $38 / M$ & Trauma & Lower leg & Descending & Coronal \& axial \\
\hline 18 & $62 / M$ & Neoplasm & Sole of foot & Descending & Coronal \& axial \\
\hline 19 & 29/M & Trauma & Lower leg & Descending & Coronal \& axial \\
\hline
\end{tabular}




\section{Fig. 1. The coronal view of multidetector-row computed tomographic images demonstrating a perforator}

(A) White arrow indicates a perforator emerging from the latissimus dorsi muscle. It is a musculocutaneous perforator branching off from the descending branch of the thoracodorsal artery and has a perpendicular course towards the overlying skin. (B) An enlarged image depicting the perforator in detail.
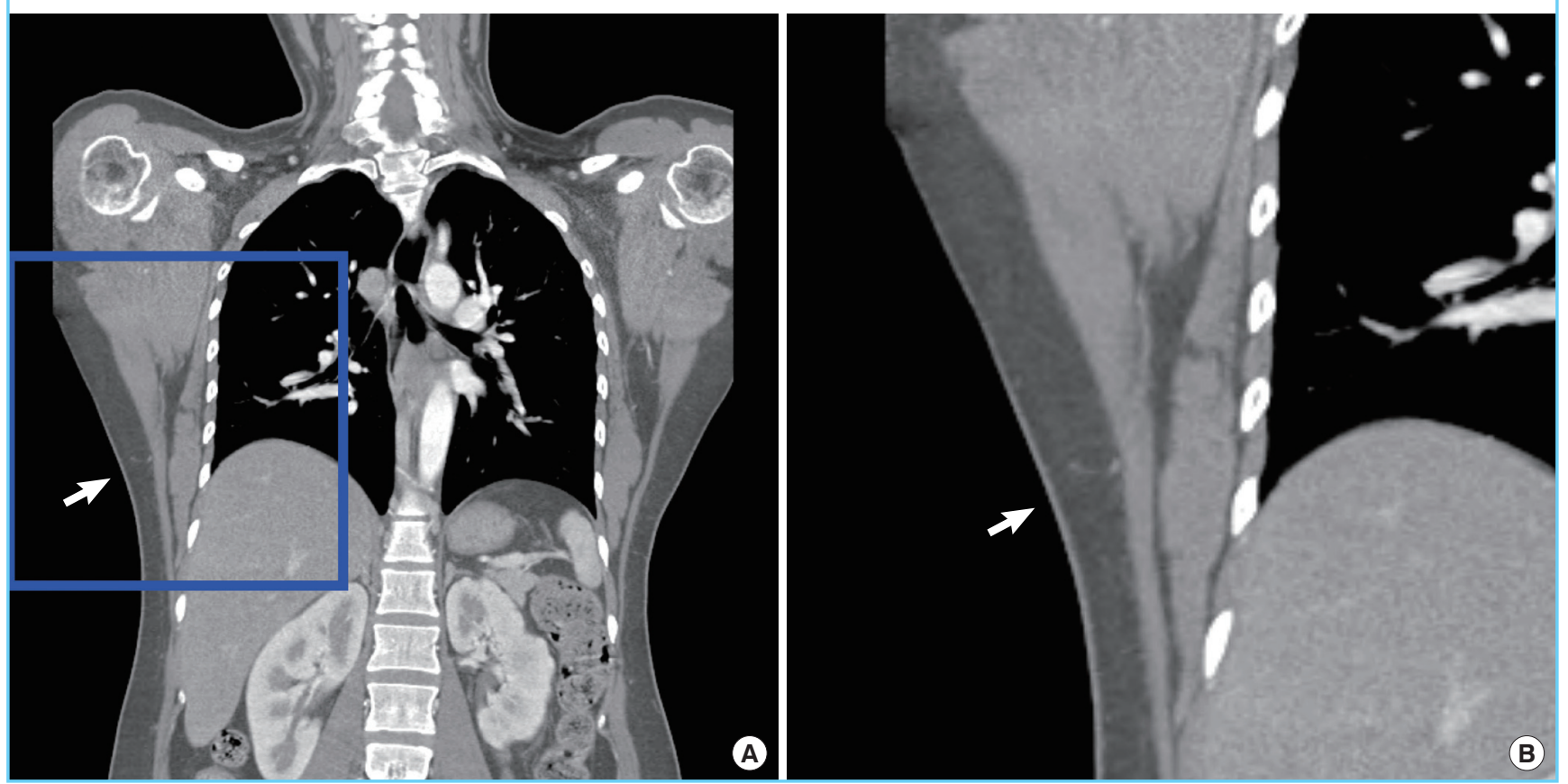

\section{Fig. 2. A 37-year-old female patient}

(A) The patient suffered a fall injury to her left tibia with exposed bone and hardware. The MDCT images of this patient are shown in Fig. 1. A chimeric flap containing the partial segment of the latissimus dorsi muscle is harvested. The pedicle of this flap originated from the descending branch of the thoracodorsal artery. (B) Flap inset was indentified 1 day after surgery.

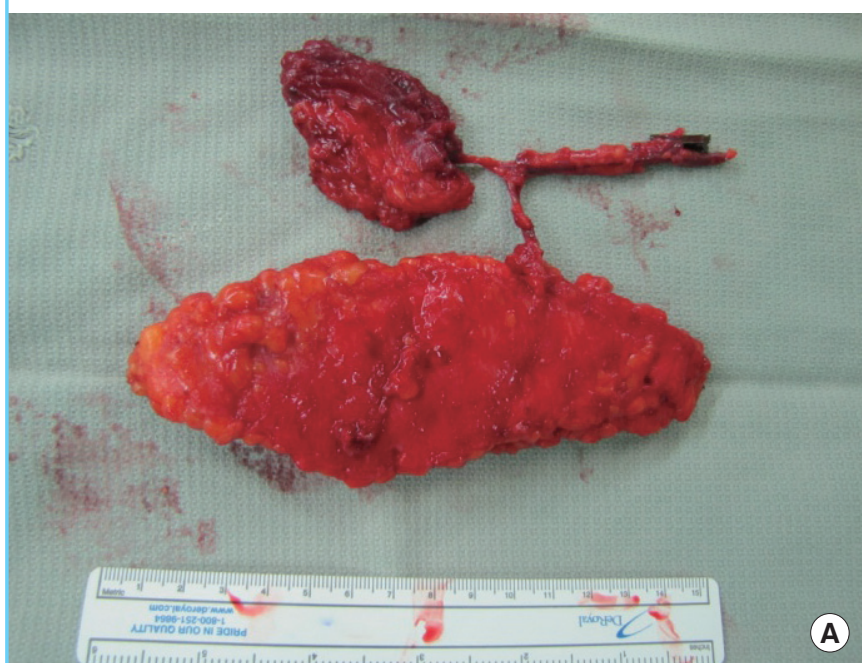

\section{DISCUSSION}

The TDAP free flap is one of the safest and most versatile flaps with a long pedicle and a large flap cutaneous territory [4]. The thoracodorsal artery, which supplies blood to the flap, is constantly bifurcated into the descending and transverse branches, which run on the deep surface of the latissimus dorsi muscle.

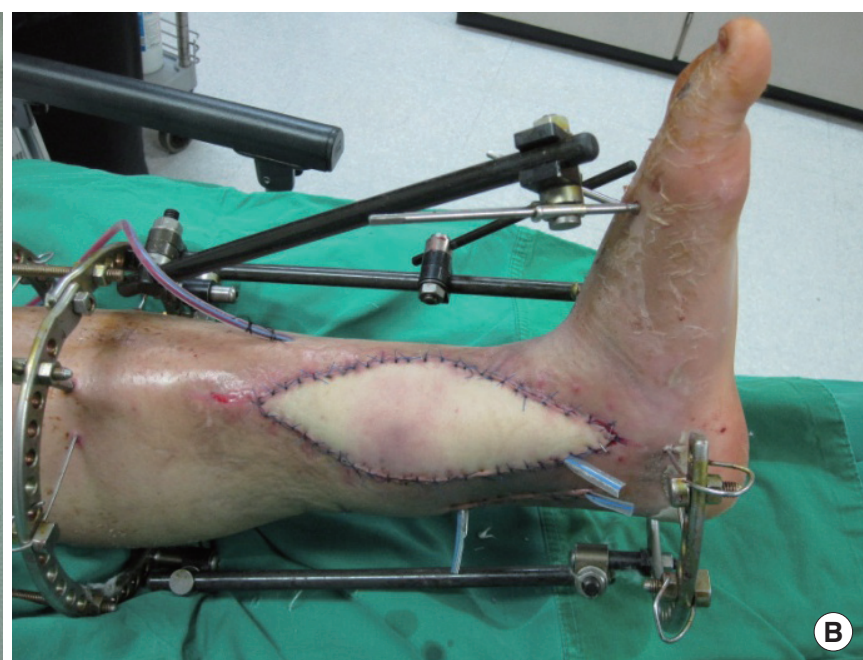

Most perforating vessels from these branches are musculocutaneous with a slightly higher percentage in the descending branch [5]. The descending branch is known to have the largest and most reliable perforators [6]. Therefore, many surgeons prefer to target perforators from the descending branch in designing the skin paddle of the TDAP free flap. This flap usually has a vertically oriented shape. However, a transverse design 


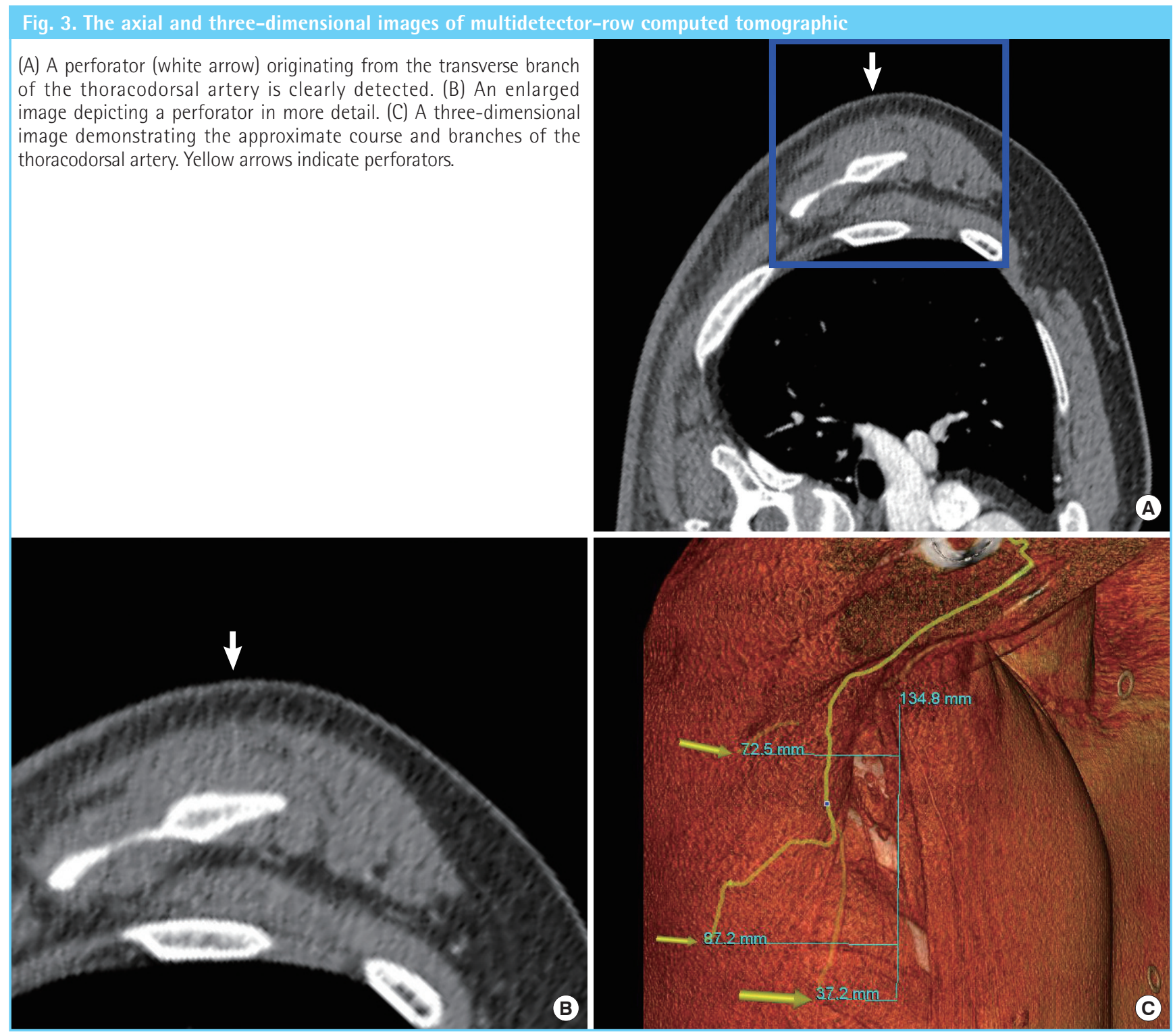

of the skin paddle could also be a better choice than a vertical design for flap versatility and donor scarring [7]. Regardless of the flap design, accurately identifying the location and course of the reliable perforators originating from both branches of the thoracodorsal artery is indispensable in the planning of the TDAP free flap. Although the vertically patterned design mostly depends on the descending branch, preoperative perforator mapping is important in ascertaining the exact individual vascular anatomy. To date, MDCT has been a very valuable tool for detecting reliable perforators. The axial view of MDCT has been very useful for searching for reliable perforators in the abdominal perforator flaps [8]. However, preoperative axial images have some limitations in detecting the best perforating vessels suitable for a vascular pedicle in TDAP free flap because of the difficulty in interpreting MDCT images of donor sites, which have smaller perforators and sparse subcutaneous tissues compared with the abdomen [1]. Thus, most surgeons have not utilized MDCT for locating thoracodorsal artery perforators during the preoperative period. Instead, the intraoperative application of hand-held Dopplers based on anatomical perforator landmarks has widely been used. However, preoperative planning of the surgery according to the Doppler findings is difficult, and intraoperative modifications of the surgical plan such as flap shape and location would be necessary. In our study, the authors thoroughly reviewed both the axial and coronal views of the MDCT images to accurately find reliable perforators. It is easier to find the perforators from the descending branch of the thoracodorsal artery in the coronal view than the axial view. On the other hand, the perforators from the transverse branch were detected in only the axial view. Most perforators detected in MDCT images were from the descending branch because the transverse branch had a small number of perforators compared 


\section{Fig. 4. Intraoperative views}

(A) Preoperatively marked perforators are confirmed during the elevation of the flap. White arrows indicate vessels from the descending branch, and the arrowhead indicates vessels from the transverse branch. (B) Thoracodorsal artery perforator flap containing a partial muscular segment is prepared for free tissue transfer. The three vessels (white arrows) to the skin paddle and muscle are from the descending branch of the thoracodorsal artery; the other one (black arrowhead) to the skin paddle is from the transverse branch. (C) Immediate postoperative view of the recipient site.

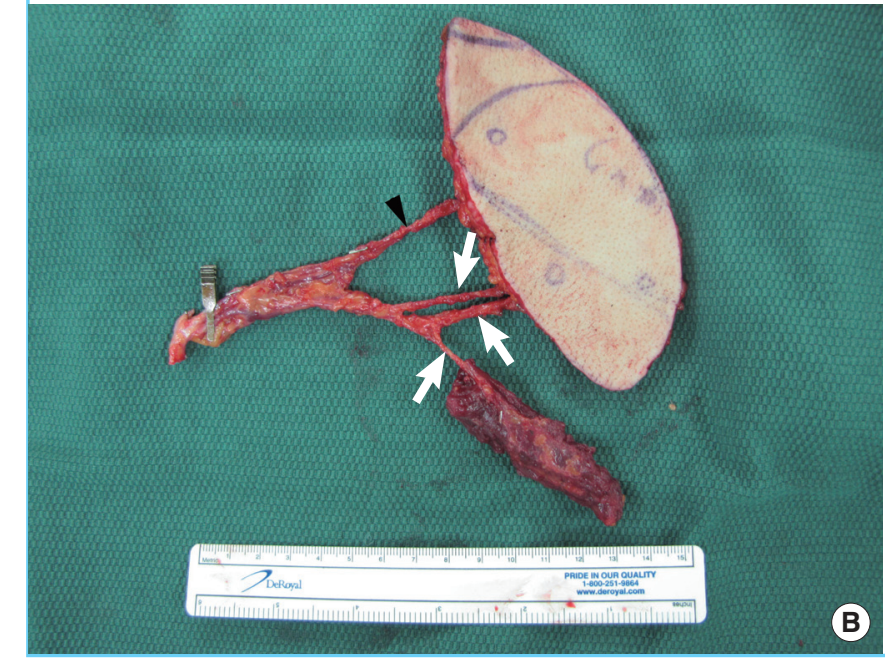

with the descending branch. Therefore, the MDCT images should always be evaluated in both the axial and coronal plane. We were able to determine the location of the reliable perforators with ease by examining both planes. Perforators not found on the axial images due to their narrow caliber or parallel course in the axial plane were detected in the coronal plane, and vice versa. The combination of the coronal and axial MDCT images enabled us to obtain more accurate information on the intramuscular course of the thoracodorsal artery perforators, which facilitated a simpler muscular dissection and shortened the operation times. In addition, reconstructing three-dimensional images by simultaneously interpreting the coronal and axial planes provided more practical images of the perforator locations and their intramuscular courses. Verifying the course of the thoracodorsal artery perforators may also help to establish preoperative plans, especially in designing and positioning the skin paddle. Moreover, with advancing CT scanning technologies that possess high-definition images and narrower slice thicknesses and intervals, our MDCT images at $1 \mathrm{~mm}$ thickness
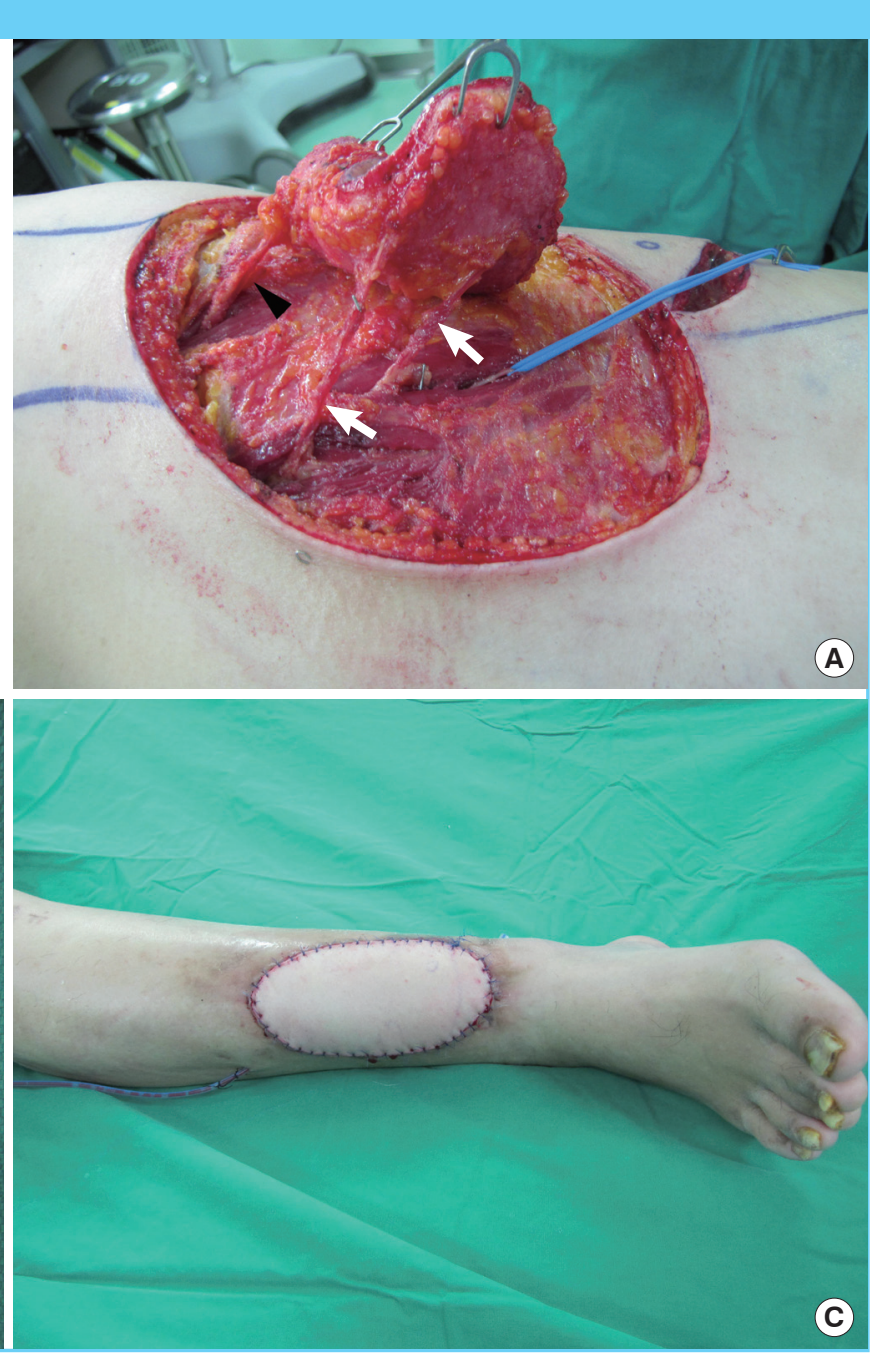

and an interval of $0.8 \mathrm{~mm}$ lowered the probability of missing reliable perforators. Thus, it is obvious that coronal MDCT images, which have not usually been examined thoroughly, also play a crucial role in searching for reliable perforating vessels, and that the assessment of both coronal and axial images will increase the specificity of MDCT.

In conclusion, the results of this study suggest that the multiple planes and 1-mm slice thickness of MDCT images can increase the probability of detecting the most reliable perforators and decrease the probability of missing available vessels. In addition, reconstructed three-dimensional images may help identify the approximate courses of source vessels and individual vascular anatomy.

\section{REFERENCES}

1. Mun GH, Kim HJ, Cha MK, et al. Impact of perforator mapping using multidetector-row computed tomographic angiography on free thoracodorsal artery perforator flap 
transfer. Plast Reconstr Surg 2008;122:1079-88.

2. Lin CT, Huang JS, Yang KC, et al. Reliability of anatomical landmarks for skin perforators of the thoracodorsal artery perforator flap. Plast Reconstr Surg 2006;118:1376-86.

3. Blondeel PN, Beyens G, Verhaeghe R, et al. Doppler flowmetry in the planning of perforator flaps. Br J Plast Surg 1998;51:202-9.

4. Saint-Cyr M, Schaverien MV, Rohrich RJ. Perforator flaps: history, controversies, physiology, anatomy, and use in reconstruction. Plast Reconstr Surg 2009; 123:132e-45e.

5. Heitmann C, Guerra A, Metzinger SW, et al. The thoracodorsal artery perforator flap: anatomic basis and clinical applica- tion. Ann Plast Surg 2003;51:23-9.

6. Guerra AB, Metzinger SE, Lund KM, et al. The thoracodorsal artery perforator flap: clinical experience and anatomic study with emphasis on harvest techniques. Plast Reconstr Surg 2004;114:32-41.

7. Lee SH, Mun GH. Transverse thoracodorsal artery perforator flaps: experience with 31 free flaps. J Plast Reconstr Aesthet Surg 2008;61:372-9.

8. Masia J, Clavero JA, Larranaga JR, et al. Multidetector-row computed tomography in the planning of abdominal perforator flaps. J Plast Reconstr Aesthet Surg 2006;59:594-9. 